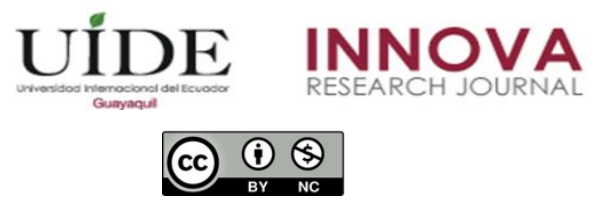

INNOVA Research Journal, ISSN 2477-9024

(Septiembre-Diciembre 2020). Vol. 5, No.3.1 pp. 76-88

DOI: https://doi.org/10.33890/innova.v5.n3.1.2020.1491

URL: http://revistas.uide.edu.ec/index.php/innova/index

Correo: innova@uide.edu.ec

\title{
La integración socioeducativa, factor clave para una mejor comprensión lectora
}

\section{Socio-educational integration, a key factor for better reading comprehension}

Aydeé de las Mercedes Rodríguez Martínez

(1) https://orcid.org/0000-0002-4770-4166

Universidad Norbert Wiener, Perú

Autor para correspondencia: aydeeroma@gmail.com

Fecha de recepción: 06 de agosto de 2020 - Fecha de aceptación: 06 de noviembre de 2020

\section{Resumen}

El presente estudio pretende integrar el contexto social en los procesos de lectura. Nace de la investigación realizada en el 2019 en la Institución Educativa La Frontera; su objetivo general es evaluar la eficacia de una propuesta pedagógica de integración socioeducativa en la mejora de la comprensión lectora de los estudiantes de sexto grado. Es una investigación aplicada, de nivel explicativo, enfoque cuantitativo y diseño cuasi experimental. La población fueron 160 estudiantes y 80 padres de estudiantes del grupo experimental. Se usó el cuestionario y como técnica, la prueba escrita. La validez de los instrumentos se realizó a través del juicio de expertos y la congruencia de jueces; la confiabilidad por medio del Coeficiente Alpha de Cronbach. Se usó la estadística descriptiva e inferencial; las pruebas de Kolmogorov-Smirnov, U de Mann-Whitney y Wilcoxon. Se diseñó e implementó "PPECLE: leer contigo, leer conmigo". Se comprobó su eficacia en los niveles inferencial $(0,048)$, crítico $(0.40)$ y de comprensión lectora general $(0,04)$, pero no en el nivel literal (0,273); al comparar los resultados en el grupo experimental (antes y después), se obtuvo una alta significancia en todos los niveles y en la comprensión lectora general $(0.00$ y 0,06$)$. Palabras claves: comprensión lectora; integración socioeducativa; lectura en familia; estrategia pedagógica.

\begin{abstract}
The present study tries to integrate the social context in the reading processes. It is born from the research carried out in 2019 at the La Frontera Educational Institution; its general objective is to evaluate the effectiveness of a pedagogical proposal of socio-educational integration in improving the reading comprehension of sixth grade students. It is an applied research, of explanatory level, quantitative approach and quasi-experimental design. The population was 160 students and 80 parents of students from the experimental group. The questionnaire was used and as a technique, the written test. The validity of the instruments was carried out through expert judgment and the consistency of judges; reliability using Cronbach's Alpha Coefficient. Descriptive and inferential statistics were used; Kolmogorov-Smirnov, U of Mann-Whitney and Wilcoxon tests. "PPECLE: read with you, read with me" was designed and implemented. Its efficacy was verified at the
\end{abstract}


inferential (0.048), critical (0.40) and general reading comprehension (0.04) levels, but not at the literal level (0.273); when comparing the results in the experimental group (before and after), a high significance was obtained at all levels and in general reading comprehension (0.00 and 0.06). Keywords: reading comprehension; socio-educational integration; family reading; pedagogical strategy.

\section{Introducción}

El presente artículo hace referencia a la tesis titulada "Integración socioeducativa como propuesta pedagógica para el mejoramiento de la comprensión lectora en sexto grado, Institución Educativa La Frontera, 2019", la cual es una investigación explicativa basado en una propuesta de intervención llevada a cabo en un contexto escolar específico. El estudio se emprende con fundamento en un problema percibido y comprobado desde dos vertientes: en primer lugar, el bajo desempeño lector concurrente de los estudiantes de la Institución (ICFES, 2016, 2017), respaldado por los datos de fuentes internacionales en el contexto latinoamericano y mundial (ICFES, 2015) a partir de los resultados de las pruebas PISA (Programa para la Evaluación Internacional de Estudiantes $)^{1}$; en segundo lugar, las poca integración de los padres a la escuela, el débil vínculo entre escuela y sociedad, en los procesos pedagógicos de este centro educativo.

El estudio se constituye como un aporte al mejoramiento del desarrollo de la comprensión lectora, reafirmando la importancia de la incorporación de la sociedad en el trabajo educativo; además, genera un aporte a la teoría existente respecto al objeto de estudio y desarrolla instrumentos de diagnóstico y rutas metodológicas que propicien otros estudios en este tópico. El objetivo general es medir el impacto de una propuesta pedagógica de integración socioeducativa, en la mejora de los procesos de comprensión lectora de los estudiantes de grado sexto ${ }^{2}$. Previo a la intervención se aplicó un test para determinar el desempeño lector de los estudiantes de manera global y por niveles (literal, inferencial y crítico textual).

"PPECLE: leer contigo, leer conmigo" es una propuesta pedagógica de integración socioeducativa que, desde el enfoque semántico comunicativo, y teniendo en cuenta los factores psicoemocionales, sociales y educativos del contexto; pretende fortalecer la lectura con estrategias propias del aprendizaje significativo, desde una mirada de reflexión, convergencia y transformación. La investigación acoge los principios de la ética de la investigación y la integridad científica, los valores de veracidad y transparencia. Se gestiona la autorización de la dirección para su realización y se tramita el consentimiento informado tanto de estudiantes como de padres de familia.

\section{Lineamientos teóricos}

\section{Perspectiva y convergencia}

\footnotetext{
${ }^{1}$ Los resultados de las Pruebas Pisa (2015), ubican a Colombia en el quinto lugar en cuanto a desempeños en lectura, siendo 9 los países evaluados. Tomado de Instituto Colombiano para la Educación Superior (ICFES, 2015). Resumen Ejecutivo Colombia en PISA 2015.

${ }^{2}$ En la tesis doctoral figuran 4 objetivos específicos, ya que además de determinar el impacto de la propuesta en la comprensión lectora general, se hace por niveles, existiendo grupo de control y grupo de intervención.
} 
La incorporación de los sujetos sociales en el aprendizaje retoma ejes teóricos como la Teoría de la Acción (Argyris y Shon, 1978), que acentúa la participación activa y consensuada de los padres de familia; la Teoría socio crítica (Habermas, 1987) que resalta la importancia de la reflexión de los individuos sobre sus realidades; la Teoría del aprendizaje social (Vygotsky, 1979), al sostener que el individuo aprende a partir de las proyecciones familiares; la Teoría de la pedagogía social (Durkeim, 1997), que asegura que los problemas educacionales pueden solventarse con la participación de la sociedad. Dentro de esta misma perspectiva, Solé y Coll (1995) ponderan la función social y socializadora de la educación escolar. Se entiende la integración socioeducativa como la participación activa y significativa de los sujetos del contexto familiar y social en los procesos de enseñanza y aprendizaje.

La comprensión lectora es un proceso complejo que se encuentra estrechamente vinculado a factores tanto textuales como contextuales y psicológicos del sujeto que lee; no solamente atañe a un problema de decodificación de un texto, sino que remite inmediatamente a categorías psicológicas, sociológicas y cognitivas que median dicho proceso. Es fundamental acentuar el enfoque epistemológico de la comprensión, asumiéndola como proceso cognitivo (Marciales, 2003).

Marciales, (2003) señala que además de los factores estructurales y textuales de la comprensión lectora, existen los intersubjetivos, intrasubjetivos, y contextuales; Saldaña (2008) y Marmolejo y Jiménez (2006), ponderan el campo de las emociones al sostener que una de las capacidades que hacen parte de la comprensión de un texto, es poder comprender las emociones de otro; Thorndike (1973) asegura, que tanto los recursos de lectura existentes en el hogar como el estatus socioeconómico de la familia, están estrechamente relacionados con la comprensión lectora; Cárdenas y Santrich (2015) le otorgan a la acción de los padres el poder de motivar y favorecer o no, el aprendizaje y la lectura de los hijos, al ser la familia el principal agente mediador; Ausubel (1995) también le otorga a los libros que hay en el hogar y la actitud de los padres el poder de incidir en la comprensión lectora. Cassany (2004) sostiene que un lector competente traspasa el componente gramatical, comprendiendo las ideas que subyacen al texto y dan cuenta del contexto del que éste emerge, sin desconocer el otro contexto, el del lector que lo devela.

Siguiendo a Cassany, Dubois (2015) hace referencia a que comprender un texto es una construcción de sentido, un proceso transaccional y de integración, en el que distintos agentes, habilidades y procesos convergen. Desde esta misma mirada, Petit (2003) enuncia que la lectura es un derecho vital, es un proceso fundamental en la formación de la subjetividad del individuo y hace parte de su esencia como ser social. Finalmente, Maslow (1968) pondera el afecto, el reconocimiento y la autoestima, como factores fundamentales para el desarrollo del ser en su Teoría de la motivación humana, siendo la lectura compartida una manera que exaltar y motivar al otro.

\section{Niveles de comprensión lectora}

En el hacer y aprender de la lengua es necesario tener en cuenta distintas habilidades, competencias y componentes. A pesar del énfasis puesto en la lectura, ésta coexiste con las otras habilidades, la escritura, la oralidad y la escucha, y todas ellas confluyen y están llenas de 
significación y cargadas de una semiótica lingüística y cultural innegables; en este estudio no existen linderos claros entre unas y otras y de alguna manera, en la propuesta pedagógica todas tienen cabida, aunque en distintas proporciones. Sobre las competencias ocurre algo similar, pues, aunque el acento está puesto en la comprensión lectora, hay dinámicas relacionadas con la interpretación y producción de textos, sin poder dibujar una clara cerca entre ellas. En armonía con Cassany (2004), la comprensión de textos tampoco puede desligar los componentes de la lengua, es decir, separar lo gramatical, de lo semántico, textual o pragmático.

Para este estudio el enfoque es semántico comunicativo y la competencia priorizada es la comunicativa. Hymes (1996) explica que la competencia comunicativa no separa lo cognitivo de lo afectivo y volitivo pues el sujeto, como ser social e individual se pone de manifiesto al comunicarse. En este sentido, al hablar de textos, hoy por hoy, es preciso tener claro que, en él, convergen múltiples factores, competencias y niveles como la sociolingüística, el análisis del discurso, el texto lingüístico y la semiótica, entre otras.

Strang (1965) describe tres niveles de comprensión: Nivel literal, nivel inferencial y nivel de comprensión crítico. Estos niveles son tenidos en cuenta por organismos que evalúan el proceso de comprensión lectora como la Organización para la Cooperación y el Desarrollo Económicos (OCDE) gestor de la prueba PISA, la Asociación Internacional para la Evaluación del Rendimiento Educativo (IEA), responsable de las pruebas PIRLS (Progress in International Reading Literacy Study o Estudio Internacional de Progreso en Comprensión Lectora), además del Instituto Colombiano para la Evaluación de la Educación (ICFES) y el Ministerio de Educación de Colombia.

Son varios los autores que hacen estas distinciones en el análisis de la comprensión lectora: Van Dijk (1996) con su teoría de las micro y macro reglas en la lectura; Ducrot (1988) con la búsqueda de sentido y los contenidos implícitos; Eco (1992) con la activación de saberes a través de las conjeturas y las intenciones ideológicas de los textos y de los autores; y, Lipman (1997) que subraya el pensamiento crítico como autocorrectivo, sensible al contexto, lleno de criterio y que conduce al juicio.

El nivel literal hace referencia a la comprensión de lo que está explícito a partir del conocimiento que se tiene del significado; el nivel inferencial se asienta en el proceso de la deducción y búsqueda de sentido más allá del significado; y, el nivel crítico intertextual se concentra en la interpretación, la toma distancia del contenido, la definición de posturas, la emisión de juicios, la permeabilidad del contexto y su relación con otros textos.

Finalmente, la estrategia pedagógica hace referencia al mecanismo diseñado para facilitar la formación del estudiante, considerando las características del sujeto que aprende, la disciplina por enseñar y el contexto socio cultural de influencia (Velazco y Mosquera, 2010). PECCLE: Leer contigo, leer conmigo, retoma los principios de la integración socioeducativa y de la lectura, que son las variables básicas de esta investigación; aprehende enfoques y lineamientos pedagógicos para su diseño e implementación y reconoce las características de los estudiantes y del contexto educativo, además de incorporar orientaciones del Ministerio de Educación de Colombia. Es una propuesta de convergencia, movilización, reflexión, transformación e innovación educativa. 


\section{Metodología}

\section{Tipo y nivel de la investigación}

De acuerdo con el propósito de la investigación, es de tipo aplicada porque utiliza acciones basadas en técnicas y procedimientos específicos, en este caso pedagógicos, para la solución eficiente de un problema. Teniendo en cuenta la naturaleza y alcance de los objetivos corresponde con el nivel explicativo, dado que se enfoca en revelar la medida en que una propuesta pedagógica de integración socioeducativa mejora la comprensión lectora en los estudiantes, lo cual implica un proceso de análisis e interpretación de resultados desde las variables y con una mirada hipotética deductiva. Desde el planteamiento del problema y la naturaleza de la información requerida en función de las variables, se trata de un estudio con enfoque cuantitativo porque se recopilan y analizar datos numéricos en relación con la variable establecida (Ñaupas, 2014; Arias, 2006; Monje, 2011; y, Fernández y Baptista, 2010)

\section{Diseño de la investigación}

Por el número de variables independientes o de estudios, es de diseño univariado al controlar o manipular una sola variable; por el método que emplea es cuasiexperimental en razón de que se pretende analizar el efecto y relación de la variable independiente con la variable dependiente (Hernández, Fernández y Baptistas, 2010); en cuanto al tipo de diseño cuasiexperimental, es un estudio antes/después, lo cual significa que aborda una medición previa a la intervención y otra posterior a ella (Monje, 2011). Finalmente, considerara un grupo de control que fundamentalmente permite analizar el impacto de la propuesta de intervención diseñada (Bisquerra, 2003).

\section{Población y muestra}

El presente estudio presenta dos poblaciones: Una población A, de característica finita, conformada por los 160 estudiantes del grado sexto de la Institución Educativa, distribuidos en cuatro aulas y una población B conformada por 80 estudiantes y sus padres o representantes (propuesta de intervención). La muestra es censal, puesto que se aborda el total de la población disponible y es de tipo no probabilístico.

\section{Técnicas e instrumentos de recolección de datos}

La evaluación planteada como una prueba pedagógica (variable dependiente, comprensión lectora) es la técnica implementada y el instrumento es el cuestionario, el cual fue validado por expertos siguiendo las indicaciones de Escurra (1998) para el análisis de sus juicios y la congruencia de los mismos. Para su validación se usó el coeficiente Alpha de Cronbach obteniendo un cálculo de 0,81 . Para analizar los resultados del test se optó por valorar el desempeño por niveles y de manera global, lo mismo que retomar la escala de desempeños establecida por el ICFES (2018), la cual los clasifica en "avanzado", "satisfactorio", "mínimo" e "insuficiente".

\section{Estadística descriptiva}


Con base en los datos cuantitativos recolectados, se presenta el procedimiento estadístico seguido para la comprobación de la hipótesis general sobre el nivel de eficacia de la propuesta, a partir de la estadística descriptiva e inferencial. Se recurre a las pruebas de Kolmogorov-Smirnov para normalidad, U de Mann-Whitney y Wilcoxon. Se usa el paquete Excel bajo ambiente Windows y el programa de SPSS.

Se determinó el desempeño inicial de comprensión lectora que presentan los estudiantes de sexto grado de la Institución Educativa La Frontera, en el nivel literal, inferencial y crítico textual a través de la prueba pedagógica pretest, a la cual se le aplicó la Prueba de normalidad (Prueba de Kolmogorov-Smirnov), concluyéndose que los datos del estudio no tienen comportamiento normal.

\section{Tabla 1}

Resultados pretest

\begin{tabular}{|c|c|c|c|c|c|c|}
\hline Nivel literal & & Avanzado & Satisfactorio & Mínimo & Insuficiente & Total \\
\hline \multirow{2}{*}{ Grupo Control } & $\mathrm{N}$ & 16 & 28 & 18 & 18 & 80 \\
\hline & $\%$ & $20,00 \%$ & $35,00 \%$ & $22,50 \%$ & $22,50 \%$ & $100,00 \%$ \\
\hline \multirow{2}{*}{ Grupo Experimental } & $\mathrm{N}$ & 21 & 20 & 28 & 11 & 80 \\
\hline & $\%$ & $26,25 \%$ & $25,00 \%$ & $35,00 \%$ & $13,75 \%$ & $100,00 \%$ \\
\hline Nivel inferencial & & Avanzado & Satisfactorio & Mínimo & Insuficiente & Total \\
\hline \multirow{2}{*}{ Grupo Control } & $\mathrm{N}$ & 20 & 31 & 20 & 9 & 80 \\
\hline & $\%$ & $25,00 \%$ & $38,75 \%$ & $25,00 \%$ & $11,25 \%$ & $100,00 \%$ \\
\hline \multirow{2}{*}{ Grupo Experimental } & $\mathrm{N}$ & 27 & 18 & 19 & 16 & 80 \\
\hline & $\%$ & $33,75 \%$ & $22,50 \%$ & $23,75 \%$ & $20,00 \%$ & $100,00 \%$ \\
\hline Nivel crítico textual & & Avanzado & Satisfactorio & Mínimo & Insuficiente & Total \\
\hline \multirow{2}{*}{ Grupo Control } & $\mathrm{N}$ & 8 & 27 & 23 & 22 & 80 \\
\hline & $\%$ & $10,00 \%$ & $33,75 \%$ & $28,75 \%$ & $27,50 \%$ & $100,00 \%$ \\
\hline \multirow{2}{*}{ Grupo Experimental } & $\mathrm{N}$ & 7 & 22 & 19 & 32 & 80 \\
\hline & $\%$ & $8,75 \%$ & $27,50 \%$ & $23,75 \%$ & $40,00 \%$ & $100,00 \%$ \\
\hline
\end{tabular}

Fuente: Elaboración Propia.

Analizando el número de aciertos en el pretest, el nivel con más baja valoración de desempeño fue el crítico-intertextual con 48,44 aciertos; el nivel literal e inferencial alcanzaron el 65,31 y 65,63\% en aciertos, respectivamente. La mayoría de los estudiantes se encuentran en un desempeño mínimo y satisfactorio.

\section{Tabla 2}

\section{Resultados Postest}

\begin{tabular}{crrrrrr}
\hline Nivel literal & & Avanzado & Satisfactorio & \multicolumn{1}{c}{ Mínimo } & Insuficiente & \multicolumn{1}{c}{ Total } \\
\hline \multirow{2}{*}{ Grupo Control } & $\mathrm{N}$ & 17 & 29 & 18 & 16 & 80 \\
& $\%$ & $21,25 \%$ & $36,25 \%$ & $22,50 \%$ & $20,00 \%$ & $100,00 \%$ \\
Grupo Experimental & $\mathrm{N}$ & 22 & 25 & 27 & 6 & 80 \\
& $\%$ & $27,50 \%$ & $31,25 \%$ & $33,75 \%$ & $7,50 \%$ & $100,00 \%$ \\
\hline
\end{tabular}




\begin{tabular}{crrrrrr}
\hline Nivel literal & & \multicolumn{1}{c}{ Avanzado } & \multicolumn{1}{c}{ Satisfactorio } & \multicolumn{1}{c}{ Mínimo } & Insuficiente & \multicolumn{1}{c}{ Total } \\
\hline \multirow{2}{*}{ Grupo Control } & $\mathrm{N}$ & 22 & 31 & 20 & 7 & 80 \\
& $\%$ & $27,50 \%$ & $38,75 \%$ & $25,00 \%$ & $8,75 \%$ & $100,00 \%$ \\
Grupo Experimental & $\mathrm{N}$ & 32 & 26 & 18 & 4 & 80 \\
Nivel crítico textual & $\%$ & $40,00 \%$ & $32,50 \%$ & $22,50 \%$ & $5,00 \%$ & $100,00 \%$ \\
Grupo Control & $\mathrm{N}$ & Avanzado & Satisfactorio & Mínimo & Insuficiente & Total \\
Grupo Experimental & $\%$ & 10 & 30 & 23 & 17 & 80 \\
& $\mathrm{~N}$ & $12,50 \%$ & $37,50 \%$ & $28,75 \%$ & $21,25 \%$ & $100,00 \%$ \\
& $\%$ & $16,25 \%$ & 33 & 18 & 16 & 80 \\
\hline
\end{tabular}

Fuente: Elaboración Propia.

En el postest el número de aciertos mejoró significativamente: En el nivel inferencial los aciertos representan el 76,56\%, en el literal el 69,69\% y en el crítico textual el 62,50\%. Sigue siendo el nivel crítico textual el que evidencia más falencias. Sin embargo, desde un cálculo diferencial se observa mayor mejora en el nivel crítico (14,06\%), seguido del nivel inferencial $(10,93) \mathrm{y}$, por último, el literal $(4,38 \%)$.

\section{Tabla 3}

Desempeño comprensión lectora general

\begin{tabular}{lrrrrrr}
\hline Pretest & & & & & & \\
\hline & & Avanzado & Satisfactorio & Mínimo & Insuficiente & Total \\
Grupo Control & $\mathrm{N}$ & 15 & 42 & 17 & 6 & 80 \\
& $\%$ & $18,75 \%$ & $52,50 \%$ & $21,25 \%$ & $7,50 \%$ & $100,00 \%$ \\
Grupo Experimental & $\mathrm{N}$ & 18 & 30 & 25 & 7 & 80 \\
Postest & $\%$ & $22,50 \%$ & $37,50 \%$ & $31,25 \%$ & $8,75 \%$ & $100,00 \%$ \\
& & & & & & \\
Grupo Control & $\mathrm{N}$ & 17 & 45 & 15 & 3 & Total \\
& $\%$ & $21,25 \%$ & $56,25 \%$ & $18,75 \%$ & $3,75 \%$ & $100,00 \%$ \\
Grupo Experimental & $\mathrm{N}$ & 26 & 39 & 13 & 2 & 80 \\
& $\%$ & $32,50 \%$ & $48,75 \%$ & $16,25 \%$ & $2,50 \%$ & $100,00 \%$ \\
\hline
\end{tabular}

Fuente: Elaboración Propia.

Desde el punto de vista de los aciertos, la comprensión lectora general en el pretest tuvo 574 aciertos, cifra que representa el 59,79\% y en el postest fueron 668 aciertos, 69,58\%, la diferencia entre dichos porcentajes es de $9,79 \%$. Es una situación muy positiva que el porcentaje de estudiantes en el nivel de mínimo e insuficiente haya bajado en ambos grupos, subiendo los otros dos; el mejoramiento es más significativo en el grupo experimental.

\section{Estadística inferencial}

Para evaluar la eficacia de la propuesta de integración socioeducativa en el mejoramiento de la comprensión lectora se realizaron dos pruebas estadísticas. La primera de ellas la de 
Wilcoxon comparando el grupo experimental antes y después de la implementación. La segunda la prueba U de Mann Whitney comparando ambos grupos antes y después.

En la Tabla 4 se observan los resultados de la Prueba de Wilcoxon: El nivel de significancia del nivel literal es de 0,006, del nivel inferencial 0,000, del nivel crítico textual 0,000 y de la comprensión lectora general 0,000.

\section{Tabla 4}

Prueba de Wilcoxon para contrastar grupo experimental, antes y después

\begin{tabular}{lcccc}
\hline & $\begin{array}{c}\text { Postest Literal } \\
\text { - Pretest Literal }\end{array}$ & $\begin{array}{c}\text { Postes } \\
\text { Inferencial - } \\
\text { Pretest } \\
\text { Inferencial }\end{array}$ & $\begin{array}{c}\text { Postes Crítico } \\
\text { - Pretest Crítico }\end{array}$ & $\begin{array}{c}\text { Postest C. } \\
\text { General - Pretest } \\
\text { C. General }\end{array}$ \\
\hline Z & $-2,762^{\mathrm{b}}$ & $-4,541^{\mathrm{b}}$ & $-4,652^{\mathrm{b}}$ & $-5,753^{\mathrm{b}}$ \\
Sig. asintót. (bilateral) &, 006 &, 000 &, 000 &, 000 \\
\hline
\end{tabular}

Fuente: Resultados prueba Wilcoxon para muestras relacionadas, SPSS (2020)

En las tablas siguientes se evidencian los resultados de la prueba U de Mann Whitney en todos y cada uno de los niveles, además de la comprensión lectora general. El índice del nivel literal es de 0,273 lo cual indica que no hay significancia pues es superior a 0,05. Para los demás sí es favorable: El nivel inferencial obtiene un 0,48, el nivel crítico textual 0,040 y de la comprensión lectora general 0,004, muy alta significancia.

\section{Tabla 5}

Prueba U de Mann-Whitney para contrastar ambos grupos, nivel literal

\begin{tabular}{lrr}
\hline & Pretest Nivel Literal & Postest Nivel Literal \\
\hline U de Mann-Whitney & 3033,000 & 2891,500 \\
W de Wilcoxon & 6273,000 & 6131,500 \\
Z &,- 591 & $-1,096$ \\
Sig. asintót. (bilateral) &, 555 &, 273 \\
\hline
\end{tabular}

Fuente: Resultados prueba U de Mann Whitney, SPSS (2020)

\section{Tabla 6}

Prueba U de Mann-Whitney para contrastar ambos grupos, nivel inferencial

\begin{tabular}{lrr} 
& Pretest N. Inferencial & Postest N Inferencial \\
\hline U de Mann-Whitney & 3168,000 & 2650,500 \\
W de Wilcoxon & 6408,000 & 5890,500 \\
Z &,- 113 & $-1,979$ \\
Sig. asintót. (bilateral) &, 910 &, 048 \\
\hline
\end{tabular}

Fuente: Resultados prueba U de Mann Whitney, SPSS (2020) 


\section{Tabla 7}

Prueba U de Mann-Whitney para contrastar ambos grupos, nivel crítico

\begin{tabular}{lrr}
\hline & Pretest N Crítico & Postest N Crítico \\
\hline U de Mann-Whitney & 3045,000 & 2624,500 \\
W de Wilcoxon & 6285,000 & 5864,500 \\
Z &,- 551 & $-2,051$ \\
Sig. asintót. (bilateral) &, 581 &, 040 \\
\hline
\end{tabular}

Fuente: Resultados prueba U de Mann Whitney, SPSS (2020)

\section{Tabla 8}

Prueba U de Mann-Whitney para contrastar ambos grupos, comprensión lectora general

\begin{tabular}{lrr}
\hline & $\begin{array}{c}\text { Pretest Comprensión } \\
\text { Lectora }\end{array}$ & $\begin{array}{c}\text { Postest Comprensión } \\
\text { Lectora }\end{array}$ \\
\hline U de Mann-Whitney & 3174,500 & 2355,000 \\
W de Wilcoxon & 6414,500 & 5595,000 \\
Z &,- 088 & $-2,917$ \\
Sig. asintót. (bilateral) &, 930 &, 004 \\
\hline
\end{tabular}

Fuente: Resultados prueba U de Mann Whitney, SPSS (2020)

\section{Discusión de resultados}

Los resultados son similares a los de Cusihualpa (2017), quien también trabajó la comprensión lectora con estudiantes de sexto grado y logró un nivel de significancia de 0.000 . En el presente estudio se obtuvo un p valor de significancia de 0.003 luego de implementado PPECLE: leer contigo, leer conmigo, quedando demostrada la eficacia de la propuesta pedagógica.

Distinto a Lastre, Chima y Padilla (2018) quienes evidenciaron una mejora significativa en los niveles literal (p: 000) e inferencial (p: 0.014 y p: 0.001) y no para el nivel crítico textual (p: 0.485 y p: 0.034), la estadística de la presente investigación, muestra un nivel de significancia de 0.48 para el nivel inferencial y 0.40 para el crítico, no habiendo p valor de significancia para el nivel literal. 2.73. En concordancia con Cassany (1999) se aprecia el desplazamiento de la gramática dentro del enfoque comunicativo, unido a la excesiva digitalización y globalización de la época postmoderna que pondera lo global en vez de lo especifico, lo general en lugar del detalle.

El estudio de Molina y Del Valle (2016) muestra que las mayores fortalezas se acentuaron en el nivel literal. De manera similar, en este estudio el nivel con más baja valoración de desempeño es el crítico textual con 18,75\%; sin embargo, contrariando a Molina y Del Valle (2016), el desempeño en el nivel inferencial supera al literal con unos porcentajes de 58,75\%, y 
$46,25 \%$ respectivamente. Estos resultados motivan la reflexión sobre el orden jerárquico de los niveles de lectura, evidenciando que éste no se puede generalizar y no siempre se desarrolla en ordena ascendente. Los procesos y factores que inciden en la lectura se encuentran y fluyen de una manera tan dinámica y compleja, que no siempre es posible identificar sus límites o campos de acción.

Izquierdo, et al. (2019) evidencia que la implicación familiar es un factor determinante sobre el desarrollo de la comprensión lectora con una significancia de 0.23. Esta investigación afirma esa estrecha relación entre lectura e integración socioeducativa (especialmente parental) al hallar un valor de 0,000 en el nivel inferencial, crítico textual y de lectura general, al comparar el grupo experimental en el pre y postest. La significancia del nivel literal entre el antes y el después fue de 0,006 .

\section{Conclusiones}

La propuesta pedagógica de integración socioeducativa "PPECLE: leer contigo, leer conmigo", mejora muy significativamente la comprensión lectora de los estudiantes de grado sexto de la Institución Educativa La Frontera, año 2019. La eficacia de la misma se sustenta en los resultados obtenidos a través de la Prueba de Wilcoxon, a partir del contraste entre los valores del pretest y el postest del grupo experimental: El nivel de significancia obtenido en los niveles inferencial, crítico y de comprensión lectora general, fue de 0.00; en el nivel literal el valor fue de 0,06 .

La propuesta pedagógica de integración socioeducativa fue eficaz teniendo en cuenta los resultados de la Prueba de U Mann Whitney al comparar los índices obtenidos por ambos grupos (control y experimental) antes y después de la intervención: El valor de significancia obtenido en la comprensión lectora general fue 0,004. Especificando los niveles, no hubo significancia en el nivel literal (0.273), pero sí en el nivel inferencial (0.048) y en el nivel crítico textual (0.040). PPECLE: Leer contigo, leer conmigo, retoma principios teóricos, pedagógicos y metodológicos desde una mirada ecléctica y transformadora. Colina (2019) resalta que la investigación socioeducativa comprende tanto el fenómeno social como el educativo, lo cual implica que no puede abordarse de manera lineal sino hermenéutica, porque en ella se producen relaciones múltiples y complejas, propias de los fenómenos educativos.

\section{Recomendaciones}

Los beneficios obtenidos podrían generalizarse a otros grupos, grados o niveles de educación, si se hace de la propuesta una práctica institucional o interinstitucional. La presencia de otros agentes además del docente, permite un mayor enriquecimiento en los procesos y resultados de comprensión lectora y constituyen un espacio propicio para que la familia se integre y comprometa; una propuesta así planteada impacta otros ámbitos como el ambiente familiar y el estado emocional. Se recomienda incorporar una entrevista con los docentes que imparten el área de lenguaje a nivel institucional, municipal o regional, para identificar prácticas pedagógicas significativas en contexto específicos que permitan enriquecer la propuesta. 
Es fundamental que la propuesta fortalezca la relación entre los estudiantes con el CRA (Biblioteca de la Institución) lo mismo que entre las familias y la biblioteca municipal, para multiplicar los beneficios y fortalecer su permanencia en el tiempo.

Pese a ponderar la lectura, no es posible desligar totalmente el proceso lector con el de escritura y otras habilidades comunicativas tal como se considera en la propuesta; de la misma manera, no es posible separar los componentes o competencias de la lengua ya que todo converge en el texto y el acto de leer. Se recomienda incorporar estrategias lúdicas, recreativas y tecnológicas propias del contexto de los estudiantes, con el fin de motivar las actividades y sacar mejor provecho. Así mismo, alejar estas propuestas de los factores académicos que impliquen nota por desempeños.

\section{Referencias bibliográficas}

Argyris, C. y Schon, D. (1978). Organizational Learning: A Theory of Action Perspective. San Francisco: Addison Wesley.

Arias, F. (2006). El proyecto de investigaciones. Guía para la elaboración. (3a ed). Caracas: Episteme

Ausubel, D. (1995). Psicología educativa. Un punto de vista cognoscitivo. México: Trillas.

Bisquerra, R. (2003). Metodología de investigación educativa. Madrid: La Muralla.

Cárdenas, R. y Santrich, E. (2015). Factores asociados a la compresión lectora en los estudiantes de noveno grado de la I.E.D. "Jesús Maestro Fe y Alegría" de Barranquilla. http://repositorio.cuc.edu.co/xmlui/bitstream/handle/11323/263/32725569.pdf?sequence $=1 \&$ isAllowed $=\mathrm{y}$

Cassany D. (2004). Los enfoques comunicativos: Elogio y crítica. Revista del Departamento de Lingüística y Literatura de la Universidad de Antioquia. https://repositori.upf.edu/bitstream/handle/10230/21223/cassany_enfoques.pdf?se

Coll, C. y Solé, I. (1993). Los profesores y la concepción constructivista. Barcelona: Graó.

Colina, A (2019). Naturaleza ontológica de la investigación socioeducativa: Elementos orientadores. https://revistas.uide.edu.ec/index.php/innova/article/view/1092/1590

Cusihualpa, J. (2017). Estrategias de aprendizaje en la comprensión lectora en estudiantes del sexto ciclo en una institución educativa, San Isidro - 2016. http://repositorio.ucv.edu.pe/bitstream/handle/20.500.12692/5240/Cusihualpa_TJI.pdf?s equence $=1 \&$ is Allowed $=\mathrm{y}$

Dubois, M. (2015). El proceso de lectura, de la teoría a la práctica. http://pdfhumanidades.com/sites/default/files/apuntes/Dubois.pdf

Ducrot, O. (1988). Polifonía y argumentación. Cali: Universidad del Valle

Durkeim, E. (1997) Educación y sociología. (6 $\sigma^{a}$ ed). Barcelona: Península.

Eco, U. (1992). Los límites de la interpretación. Recuperado de https://monoskop.org/images/0/0f/Eco_Umberto_Los_Limites_de_la_Interpretacion_19 92.pdf

Escurra, L. (1998). Cuantificación de la validez de contenido por juicio de expertos. Recuperado de https://dialnet.unirioja.es/servlet/articulo?codigo=6123333

Habermas, J. (1987). Teoría y práctica; teoría y praxis. Estudios de filosofía social. Madrid: Tecnos. 
Hernández, R., Fernández, C. y Baptista, P. (2010). Metodología de la investigación. (5a ed). México: McGraw-Hill Interamericana.

Hymes, D. (1996). Acerca de la competencia comunicativa. Revista Forma y Función (9). Bogotá: Universidad Nacional de Colombia.

Instituto Colombiano para la Educación Superior (ICFES, 2015). Resumen Ejecutivo Colombia en PISA

2015. https://www.icfes.gov.co/documents/20143/237304/Informe\%20resumen\%20ejecutivo\% 20colombia\%20en\%20pisa\%202015.pdf

Instituto Colombiano para la Evaluación de la Educación (ICFES, 2016). Informe de resultados. Pruebas Saber Tercero, Quinto y Noveno, IE La Frontera, Villa del Rosario, Norte de Santander.

Instituto Colombiano para la Evaluación de la Educación (ICFES, 2017). Índice Sintético de Calidad Educativa de la Institución Educativa La Frontera IE La Frontera, Villa del Rosario, Norte de Santander.

Instituto Colombiano para la Evaluación de la Educación (ICFES, 2018). Guía de uso e interpretación de resultados. http://www.icfesinteractivo.gov.co/resultados-saber2016web/resources/docs/Guia_Reporte_Resultados_Saber359_2018.pdf

Izquierdo, T., Sánchez M. y López, E. (2019). Determinantes del entorno familiar en el fomento del hábito lector del alumnado de Educación Primaria. Estudios sobre Educación 36, 157179. doi: https://doi.org/10.15581/004.36.157-179

Lastre, K., Chimá, F. y Padilla, A. (2018). Efectos de la lectura en voz alta en la compresión lectora de estudiantes de primaria. Revista Encuentros, 16(01), 11-22. doi: http://dx.doi.org/10.15665/.v16i01.945

Lipman, M. (1997). Pensamiento complejo y educación. Madrid: Ediciones de La Torre.

Marmolejo, F. y Jiménez, A. (2006). Inferencias, modelos de situaciones y emociones en textos narrativos. El caso de los niños en edad preescolar. Revista Intercontinental de Psicología y Educación, 8(2), 93-138. https://www.redalyc.org/articulo.oa?id=802/80280207

Maslow, A. (1968). El hombre autorrealizado, hacia una psicología del ser. Buenos Aires: Troquel.

Marciales, G. (2003). Pensamiento crítico: diferencias en estudiantes universitarios en el tipo de creencias, estrategias e inferencias en la lectura crítica de textos. Recuperado de http://webs.ucm.es/BUCM/tesis//edu/ucm-t26704.pdf

Monje, C. (2011). Metodología de la investigación cualitativa y cuantitativa. Guía didáctica. Neiva: Universidad Surcolombiana.

Molina, T. y Del Valle, B. (2016). Fortalecimiento de la lectura mediante la interacción familiar: Programa PROLECS-UNA. http://www.redalyc.org/articulo.oa? id=35649692010

Naupas, H. (2014). Metodología de la investigación. Cuantitativa-cualitativa y redacción de la tesis. (4a ed). Bogotá: Ediciones de la U.

Petit, M. (2003). La lectura, íntima y compartida. https://docplayer.es/7089709-La-lectura-intimay-compartida.html

Saldaña, D. (2008). Teoría de la mente y lectura en las personas con trastornos del espectro autista: hipótesis para una relación compleja. Revista de Logopedia, Foniatría y Audiología, 28(2), 117-125. https://doi.org/10.1016/S0214-4603(08)70050-0

Strang, R. (1965), Procesos del aprendizaje infantil, Buenos Aires, Paidós.

Smith, C. (1989). La enseñanza de la lecto-escritura: un enfoque interactivo. Madrid: Aprendizaje Visor. 
Solé, I. (1996). Estrategias de comprensión de la lectura. Barcelona: Graó.

Thorndike, R. (1973). La comprensión lectora: Educación en quince países. Nueva York: John Wiley.

Van Dijk, T. (1996). Estructura y funciones del discurso. Traducido por Gann y Mur. http://www.discursos.org/oldbooks/Teun\%20A\%20van\%20Dijk\%20$\%$ 20Las\%20Estructuras\%20y\%20Funciones\%20del\%20Discurso.pdf

Velazco, M. y Mosquera, F. (2010). Estrategias Didácticas para el Aprendizaje Colaborativo. http://acreditacion.udistrital.edu.co/flexibilidad/estrategias_didacticas_aprendizaje_colab orativo.pdf

Vygotsky, L. (1.979). El desarrollo de los procesos psicológicos superiores. Barcelona: Grijalbo. 